\title{
Mamíferos coletados em Pedra Branca, Município de Paraty, Rio de Janeiro, Brasil
}

\author{
Sergio Maia Vaz
}

Departamento de Vertebrados, Seção de Mamíferos, Museu Nacional. Quinta da Boa Vista, 20940-040 Rio de Janeiro, Rio de Janeiro, Brasil. E-mail: smvaz@mn.ufrj.br

\begin{abstract}
Mammals collected in Pedra Branca, Municipality of Paraty, State of Rio de Janeiro, Brazil. A survey of wild mammals species from the region of Pedra Branca, municipality of Paraty, Rio de Janeiro, Brazil, housed in Museu Nacional collection, is presented. This material was assembled during the 1940's, and include thirty-three species: six opossums, two xenarthrans, four primates, four carnivores, two artiodactyls and fifteen rodents.
\end{abstract}

KEY WORDS. Mastofauna, Museu Nacional, Serra do Mar, survey.

RESUMO. Um levantamento de espécies de mamíferos silvestres da região de Pedra Branca, município de Paraty, Rio de Janeiro, Brasil, depositado na coleção do Museu Nacional, é apresentado. Esse material foi reunido na década de 1940 e inclui trinta e três espécies: seis marsupiais, dois xenartros, quatro primatas, quatro carnívoros, dois artiodáctilos e quinze roedores.

PALAVRAS CHAVE. Levantamento, mastofauna, Museu Nacional, Serra do Mar.

Em 1944, Herbert Franzoni Berla, ornitólogo do Museu Nacional, publicou uma lista de aves obtidas na região de Pedra Branca, município de Paraty, baseada em duas excursões feitas no ano de 1941 (Berla 1944).

Exame realizado no acervo da seção de mamíferos do Museu Nacional, permitiu a localização de um considerável número de espécimes procedentes do mesmo local, inclusive de alguns indivíduos coletados por H.F. Berla.

Embora essa coleção mastozoológica tenha sido formada há mais de 50 anos, ela nunca foi objeto de maiores estudos.

\section{MATERIAL E MÉTODOS}

Pedra Branca é a denominação de uma extensa área $\left(23^{\circ} 09^{\prime}\right.$ $23^{\circ} 13^{\prime}$ S; $44^{\circ} 45^{\prime}-44^{\circ} 48^{\prime} \mathrm{W}$ ), localizada no município de Paraty, sul do estado do Rio de Janeiro. Trata-se de uma região bastante acidentada, encravada na Serra do Mar, cuja altitude varia de 100 a 1.545 m (Ibge 1974). A área é revestida pela Floresta Ombrófila Densa, formações Submontana e Montana (IbAMA 1989, Oliveira 1995). A cobertura florestal encontra-se relativamente bem preservada. Acima da cota altimétrica de $500 \mathrm{~m}$ o lugar fica inserido nos limites do Parque Nacional da Serra da Bocaina.

O presente estudo baseia-se no material mastozoológico proveniente de Pedra Branca (Paraty) que se encontra depositado no Museu Nacional, Universidade Federal do Rio de Janeiro (MNRJ).

A identificação das espécies foi feita com o auxílio dos estudos de Vieira $(1944,1950,1955)$, Moojen (1952), Cabrera
(1958, 1961), Wetzel \& Mondolfi (1979), Wetzel \& Avila Pires (1980), Emmons \& Feer (1990), Hershrovitz $(1944,1992)$, Patton \& da Silva (1997), Musser et al. (1998), Pereira et al. (2001) e também através da comparação com outros exemplares conservados na mencionada instituição.

O material listado foi disposto de acordo com a ordem sistemática proposta por WiLSON \& REEDER (1993).

Entre setembro de 1999 e fevereiro de 2000, foram realizadas visitas à Paraty com o propósito de levantar, junto a população local, informações sobre a mastofauna regional. Não foram feitas coletas de espécimes por não haver autorização do IвAма para isso e também por não constituir o objetivo principal desse estudo. Pedra Branca foi alvo de visita apenas para se ter uma idéia do estado atual de conservação da cobertura florestal e da presença humana no lugar.

\section{RESULTADOS}

A coleção de mamíferos de Pedra Branca foi organizada entre 1941 e 1949. Ela foi formada a partir de coletas realizadas por Herbert Berla, Abel Chagas de Oliveira e moradores locais (José Silvestri, Ervin, Orlando, Floripes, Hélio e José Rita).

As informações contidas nas etiquetas de campo dos espécimes se resumem a procedência, sexo, data de captura e nome do coletor, não havendo menção a medidas corporais.

Investigações realizadas na coleção do Museu Nacional possibilitaram a localização de 517 indivíduos. Esse material representa 6 ordens, 17 famílias, 31 gêneros e 33 espécies (Tab. I).

Revista Brasileira de Zoologia 22 (4): 1164-1169, dezembro 2005 
Tabela I. Lista das espécies de mamíferos procedentes de Pedra Branca.

\begin{tabular}{|c|c|c|c|c|c|}
\hline Táxons & Nomes comuns & Machos & Fêmeas & Sexo indeterminado & Total \\
\hline \multicolumn{6}{|l|}{ Didelphimorphia } \\
\hline \multicolumn{6}{|l|}{ Didelphidae } \\
\hline Caluromys philander & & 1 & 1 & & 2 \\
\hline Gracilinanus microtarsus & & 1 & & & 1 \\
\hline Metachirus nudicaudatus & & & 1 & & 1 \\
\hline Monodelphis iheringi & & 2 & 1 & 1 & 4 \\
\hline Monodelphis scalops & & 1 & & & 1 \\
\hline Philander frenata & Cuíca, goitica & 13 & 7 & 1 & 21 \\
\hline \multicolumn{6}{|l|}{ Xenarthra } \\
\hline \multicolumn{6}{|l|}{ Bradypodidae } \\
\hline Bradypus variegatus & Preguiça & 4 & 3 & 4 & 11 \\
\hline \multicolumn{6}{|l|}{ Dasypodidae } \\
\hline Dasypus novemcinctus & Tatu galinha & 1 & & & 1 \\
\hline \multicolumn{6}{|l|}{ Primates } \\
\hline \multicolumn{6}{|l|}{ Callitrichidae } \\
\hline Callithrix aurita & Mico, macaco-chuin & 1 & & & 1 \\
\hline \multicolumn{6}{|l|}{ Atelidae } \\
\hline Alouatta guariba clamitans & Barbado & & 1 & & 1 \\
\hline Brachyteles arachnoides & Muriquí, mono & 2 & 2 & 2 & 6 \\
\hline \multicolumn{6}{|l|}{ Cebidae } \\
\hline Cebus nigritus nigritus & Macaco-topete, macaco & 1 & 2 & & 3 \\
\hline \multicolumn{6}{|l|}{ Carnivora } \\
\hline \multicolumn{6}{|l|}{ Felidae } \\
\hline Leopardus tigrinus & Gato do mato & 1 & & & 1 \\
\hline \multicolumn{6}{|l|}{ Mustelidae } \\
\hline Eira barbara & Irara & 1 & & & 1 \\
\hline Galictis cuja & Furão, guaxinim & & 3 & & 3 \\
\hline \multicolumn{6}{|l|}{ Procyonidae } \\
\hline Nasua nasua & Coatí & 5 & 4 & 2 & 11 \\
\hline \multicolumn{6}{|l|}{ Artiodactyla } \\
\hline \multicolumn{6}{|l|}{ Tayassuidae } \\
\hline Tayassu pecari & Queixada & 4 & 6 & & 10 \\
\hline Pecari tajacu & Cateto & 1 & 2 & & 3 \\
\hline \multicolumn{6}{|l|}{ Rodentia } \\
\hline \multicolumn{6}{|l|}{ Sciuridae } \\
\hline Sciurus aestuans & Caxinguelo & 6 & 4 & & 10 \\
\hline \multicolumn{6}{|l|}{ Muridae } \\
\hline Akodon cursor & Rato & 97 & 42 & 2 & 141 \\
\hline Delomys dorsalis & Rato & 15 & 18 & 2 & 35 \\
\hline Nectomys squamipes & Rato & 24 & 12 & & 36 \\
\hline Oligoryzomys nigripes & Rato & 4 & 4 & & 8 \\
\hline Oryzomys ratticeps & Rato & 11 & 7 & 3 & 21 \\
\hline Oryzomys russatus & Rato & 3 & 9 & & 12 \\
\hline Oxymycterus dasytrichus & Rato & 6 & 4 & 1 & 11 \\
\hline Thaptomys nigrita & Rato & 1 & 4 & 1 & 6 \\
\hline
\end{tabular}


Tabela I.Continuação.

\begin{tabular}{|c|c|c|c|c|c|}
\hline Táxons & Nomes comuns & Machos & Fêmeas & Sexo indeterminado & Total \\
\hline \multicolumn{6}{|l|}{ Erethizontidae } \\
\hline Sphiggurus villosus & Ouriço & 4 & 4 & 2 & 10 \\
\hline \multicolumn{6}{|l|}{ Hydrochaeridae } \\
\hline Hydrochaeris hydrochaeris & Capivara & & & 1 & 1 \\
\hline \multicolumn{6}{|l|}{ Dasyproctidae } \\
\hline Dasyprocta leporina & Cutia & 3 & 2 & 1 & 6 \\
\hline \multicolumn{6}{|l|}{ Cuniculidae } \\
\hline Cuniculus paca & Paca & 2 & 4 & & 6 \\
\hline \multicolumn{6}{|l|}{ Echimyidae } \\
\hline Kannabateomys amblyonyx & Rato taquara, rato bambu & & 1 & & 1 \\
\hline Trinomys dimidiatus & Rato & 69 & 57 & 5 & 131 \\
\hline
\end{tabular}

\section{Didelphimorphia Didelphidae}

\section{Caluromys philander (Linnaeus, 1758)}

Material examinado. Dois exemplares (MNRJ 6100, 8217).

\section{Gracilinanus microtarsus (Wagner, 1842)}

Material examinado. Um exemplar (MNRJ 8205).

Observações. Endêmica do Brasil, a espécie ocorre do estado de Minas Gerais ao Rio Grande do Sul (EMmons \& FeER 1990). No estado do Rio de Janeiro, o MNRJ possui espécimes colecionados em Teresópolis, Cachoeiras de Macacu e Angra dos Reis.

\section{Metachirus nudicaudatus (E. Geoffroy, 1803)}

Material examinado. Um exemplar (MNRJ 8219).

\section{Monodelphis iheringi (Thomas, 1888)}

Material examinado. Quatro exemplares (MNRJ 6102, 6221, 6500, 8203).

Observações. Endêmica do Brasil, a espécie ocorre do estado do Espírito Santo ao Rio Grande do Sul. No estado do Rio de Janeiro, o MNRJ possui espécimes colecionados em Teresópolis, Rio de Janeiro e Angra dos Reis.

\section{Monodelphis scalops (Thomas, 1888)}

Material examinado. Um exemplar (MNRJ 6419).

Observações. Endêmica do Brasil, a espécie ocorre do estado do Espírito Santo à Santa Catarina. No estado do Rio de Janeiro, o MNRJ possui espécimes colecionados em Teresópolis, Petrópolis e Rio de Janeiro.

\section{Philander frenata (Olfers, 1818)}

Material examinado. Vinte e um exemplares (MNRJ 6098, $6219,6220,6242,6243,6420,6421,7715,7716,8218,8220-$ $8228,8445,8446)$

Observações: Endêmica do Brasil, a espécie ocorre na região costeira, entre o norte da Bahia e o Rio Grande do Sul, como também nos estados de Minas Gerais e Goiás (PATTON \&
DA Silva 1997). No estado do Rio de Janeiro, o MNRJ possui espécimes colecionados em Casimiro de Abreu, Teresópolis, Petrópolis, Rio de Janeiro, Nova Iguaçu, Mangaratiba, Rio Claro e Itatiaia.

\section{Xenarthra}

\section{Bradypodidae}

\section{Bradypus variegatus Schinz, 1825}

Material examinado. Onze exemplares (MNRJ 5643, 5650, 6103, 6702, 8450, 8451, 7608, 7609, 23893, 23894, 46516).

\section{Dasypodidae}

\section{Dasypus novemcinctus Linnaeus, 1758}

Material examinado. Um exemplar (MNRJ 8464).

\section{Primates \\ Callitrichidae}

\section{Callithrix aurita (E. Geoffroy, 1812)}

Material examinado. Um exemplar (MNRJ 6101)

Observações. Endêmica do Brasil, a espécie ocorre nos estados de Minas Gerais, Rio de Janeiro e São Paulo. No estado do Rio de Janeiro, o MNRJ possui espécimes colecionados em Teresópolis, Três Rios, Angra dos Reis e Itatiaia. Além dos municípios citados, CoImbra-Filho (1991), fez menção a ocorrência da espécie em Nova Friburgo, Santa Maria Madalena, Campos, Petrópolis e Cantagalo.

\section{Atelidae}

\section{Alouatta guariba clamitans Cabrera, 1940}

Material examinado. Um exemplar (MNRJ 8452).

\section{Brachyteles arachnoides (E. Geoffroy, 1806)}

Material examinado. Seis exemplares (MNRJ 5651, 6699, 7724, 8513-8515). 
Observações. Endêmica do Brasil, AguirRe (1971), levantou que a espécie se distribuía do sul da Bahia ao norte do Paraná. Apesar de ser considerado um animal em extinção, o muriquí ou mono ainda está presente no município de Paraty, inclusive na região de Pedra Branca. No estado do Rio de Janeiro, o MNRJ possui espécimes colecionados em Teresópolis e Angra dos Reis (Mambucaba). Ávila-Pires \& Gouveia (1977) reportaram três exemplares no Parque Nacional do Itatiaia. VAZ (1998) relatou a ocorrência da espécie no Parque Estadual do Desengano (municípios de Santa Maria Madalena, São Fidélis e Campos dos Goytacazes).

\section{Cebidae}

\section{Cebus nigritus nigritus (Goldfuss, 1809)} 23364).

Material examinado. Três exemplares (MNRJ 8341, 8342,

Observações. Endêmica do Brasil, a subespécie ocorre do estado do Rio de Janeiro ao Rio Grande do Sul. No estado do Rio de Janeiro, o MNRJ possui espécimes colecionados em Teresópolis, Rio de Janeiro, Nova Friburgo, Angra dos Reis e Itatiaia.

\section{Carnivora \\ Felidae}

\section{Leopardus tigrinus (Schreber, 1775)}

Material examinado. Um exemplar (MNRJ 25650).

\section{Mustelidae}

Eira barbara (Linnaeus, 1758)

Material examinado. Um exemplar (MNRJ 5649).

\section{Galictis cuja (Molina, 1782)}

Material examinado. Três exemplares (MNRJ 8236-8238).

\section{Procyonidae}

\section{Nasua nasua (Linnaeus, 1766)}

Material examinado. Onze exemplares (MNRJ 6695, 6697, 7658, 24798-24801, 32392-32394, 32430).

Observações. O gênero foi revisado por DeCKer (1991). A espécie é comum na região de Pedra Branca.

\section{Artiodactyla Tayassuidae}

\section{Tayassu pecari (Link, 1795)}

Material examinado. Dez exemplares (MNRJ 8454-8456, 8462, 8463, 37017-37020, 37065).

Observações. O queixada praticamente desapareceu do estado do Rio de Janeiro, exceto da região montanhosa localizada nos municípios de Angra dos Reis e Paraty. Segundo depoimentos de moradores locais, em Pedra Branca, ele já não é avistado com tanta freqüência como era há 25 anos.

\section{Pecari tajacu (Linnaeus, 1758)}

Material examinado. Três exemplares (MNRJ 5646, 5647, 8453).

Observações. Essa espécie de porco do mato é bastante conhecida e caçada na região de Pedra Branca.

\section{Rodentia \\ Sciuridae}

\section{Sciurus aestuans Linnaeus, 1766}

Material examinado. Dez exemplares (MNRJ 4101, 4108, $6099,6218,6417,6418,8442-8444,29291)$.

\section{Muridae}

\section{Akodon cursor (Winge, 1887)}

Material examinado. Cento e quarenta e um exemplares (MNRJ 6190-6197, 6199-6204, 6206, 6212, 6214, 6217, 6223, 6225, 6227, 6300-6305, 6307-6314, 6316-6320, 6355-6363, 6365, 6367-6385, 6387-6392, 6401, 6403-6410, 6412-6416, 8159-8193, 8426-8441, 46515).

Observações: Moojen (1952) relatou que em Paraty, a reprodução da espécie "se faz francamente entre agosto e março".

\section{Delomys dorsalis (Hensel, 1872)}

Material examinado. Trinta e cinco exemplares (MNRJ 6198, 6207, 6211, 6213, 6216, 6224, 6226, 6290-6292, 7705, 8146-8154, 8407-8409, 8411-8416, 8418-8420, 32850).

Observações. O gênero foi revisado por Voss (1993) que reconheceu duas espécies (Delomys dorsalis e D. sublineatus), ambas ocorrendo no estado do Rio de Janeiro.

\section{Nectomys squamipes (Brants, 1827)}

Material examinado. Trinta e seis exemplares (MNRJ 6205, 6276-6280, 6282-6289, 6306, 6394, 6395, 7707, 8140-8145, 8206-8209, 8211-8214, 8395-8398).

\section{Oligoryzomys nigripes (Olfers, 1818)}

Material examinado. Oito exemplares (MNRJ 6323-6330).

\section{Oryzomys ratticeps (Hensel, 1873)}

Material examinado. Vinte e um exemplares (MNRJ 6208, $6209,6215,6222,6281,6396-6400,8210,8215$, 8216, 8399-8406).

\section{Oryzomys russatus (Wagner, 1848)}

Material examinado. Doze exemplares (MNRJ 6210, 6321, 6322, 8155-8158, 8421-8425).

\section{Oxymycterus dasytrichus (Schinz, 1821)}

Material examinado. Onze exemplares (MNRJ 6189, 62936299, 7702, 7703, 7706).

Observações: Endêmica do Brasil, ocorre nos estados da Bahia, Espírito Santo, Minas Gerais, Rio de Janeiro e São Paulo. No estado do Rio de Janeiro, o MNRJ possui espécimes colecionados em Teresópolis, Rio de Janeiro, Duque de Caxias, Nova Iguaçu, Mangaratiba, Rio Claro e Itatiaia. 
Thaptomys nigrita (Lichtenstein, 1829)

Material examinado. Seis exemplares (MNRJ 6315, 6364, $6366,6386,6402,32851)$.

\section{Erethizontidae}

\section{Sphiggurus villosus (F. Cuvier, 1822)}

Material examinado. Dez exemplares (MNRJ 8239-8243, 46517-46521).

Observações. Endêmica do Brasil, a espécie ocorre do estado de Minas Gerais ao Rio Grande do Sul (Woods 1993). No estado do Rio de Janeiro, o MNRJ possui espécimes colecionados em Casimiro de Abreu, Teresópolis, Petrópolis, Sumidouro, Maricá, Mangaratiba e Angra dos Reis.

\section{Hydrochaeridae}

\section{Hydrochaeris hydrochaeris (Linnaeus, 1766)}

Material examinado. Um exemplar (MNRJ 7663).

\section{Dasyproctidae}

\section{Dasyprocta leporina (Linnaeus, 1758)}

Material examinado. Seis exemplares (MNRJ 5662, 6694, 7718, 7719, 8447, 8448).

\section{Cuniculidae}

\section{Cuniculus paca (Linnaeus, 1766)}

Material examinado. Seis exemplares (MNRJ 8449, 84578461).

\section{Echimyidae}

\section{Kannabateomys amblyonyx (Wagner, 1845)}

Material examinado. Um exemplar (MNRJ 31576).

\section{Trinomys dimidiatus (Günther, 1877)}

Material examinado. Cento e trinta e um exemplares (MNRJ 4001-4004, 6119, 6121-6148, 6154-6166, 6255-6275, 6422, 7700, 7701, 8104-8138, 8202, 8371-8394, 11333).

Observações. Endêmica do Brasil, a espécie ocorre nos estados do Rio de Janeiro e São Paulo. No Estado do Rio de Janeiro, o MNRJ possui espécimes colecionados em Teresópolis, Guapimirim, Rio de Janeiro, Nova Iguaçu, Rio Claro e Angra dos Reis. Ávila-Pires \& Gouvea (1977) fizeram menção a ocorrência da espécie no Parque Nacional do Itatiaia.

\section{CONCLUSÕES}

O material obtido em Pedra Branca constitui a mais importante coleção de mamíferos já reunida na região Sul Fluminense. Das 33 espécies relacionadas, 10 (Gracilinanus microtarsus, Monodelphis iheringi, Monodelphis scalops, Philander frenata, Callithrix aurita, Brachyteles arachnoides, Cebus nigritus, Oxymycterus dasytrichus, Sphiggurus villosus, Trinomys dimidiatus) são endêmicas do Brasil; três (Callithrix aurita, Brachyteles arachnoides, Leopardus tigrinus) fazem parte da Lista das Espécies da Fauna Brasileira Ameaçadas de Extinção (IвAмA 2003).

Possivelmente a maioria das espécies colecionadas, na década de 1940, ainda ocorre em Pedra Branca. Esse ponto de vista é baseado no estado de conservação da cobertura florestal, na restrição de acesso à região causada pela acidentabilidade das terras, no fato daquela área estar incluída parcialmente nos limites do Parque Nacional da Serra da Bocaina e devido a baixa densidade humana presente no lugar.

\section{AGRADECIMENTOS}

À bibliotecária Solange P. Lyrio Gomes pela ajuda na preparação da tabela. Aos senhores Zaqueu Figueira Gama, Waldimiro e Joir pelas informações prestadas.

\section{REFERÊNCIAS BIBLIOGRÁFICAS}

AguiRre, A.C. 1971. O Mono Brachyteles arachnoides (E. Geoffroy). Situação atual da espécie no Brasil. Rio de Janeiro, Academia Brasileira de Ciências, 53p.

Ávila-Pires, F.D. \& E. Gouvea. 1977. Mamíferos do Parque Nacional do Itatiaia. Boletim do Museu Nacional, Nova Série Zoologia, Rio de Janeiro, 291: 1-29.

BerLa, H.F. 1944. Lista das aves colecionadas em Pedra Branca, município de Paratí, Estado do Rio de Janeiro, com algumas notas sobre sua biologia. Boletim do Museu Nacional, Nova Série Zoologia, Rio de Janeiro, 18: 1-21.

Cabrera, A. 1958. Catalogo de los mamíferos de América del Sur. Revista del Museo Argentino de Ciencias Naturales "Bernardino Rivadavia", Buenos Aires, 4 (1): 1-307.

Cabrera, A. 1961. Catalogo de los mamíferos de América del Sur. Revista del Museo Argentino de Ciencias Naturales "Bernardino Rivadavia", Buenos Aires, 4 (2): 309-732.

Cormbra-Filho, A.F. 1991. Apontamentos sobre Callithrix aurita (E. Geoffroy, 1812), um sagüi pouco conhecido, p. 145-158. In: A.B. Rylands \& A.T. Bernardes (Eds). A Primatologia no Brasil - 3. Belo Horizonte, Sociedade Brasileira de Primatologia, Fundação Biodiversitas, 459p.

Decker, D.M. 1991. Systematics of the coatis, genus Nasua (Mammalia, Procyonidae). Proceedings of Biological Society of Washington 104 (2): 370-386.

EMmons, L.H. \& F. FeER. 1990. Neotropical Rainforest Mammals: A field guide. Chicago, The University of Chicago Press, $\mathrm{XIV}+281 \mathrm{p}$.

Hershrovitz, P. 1944. A systematic review of the Neotropical water rats of the genus Nectomys (Cricetinae). Miscellaneous Publications, Museum of Zoology, University of Michingan, 58: 1-88.

Hershkovitz, P. 1992. The South American gracile mouse opossums, genus Gracilinanus Gardner and Creighton, 1989 (Marmosidae, Marsupialia): A taxonomic review with notes on general morphology and relationships. Fieldiana, 
Zoology, New Series, Chicago, 70: 1-56.

Iвама. 1989. Unidades de Conservação do Brasil. Brasília, Instituto Brasileiro do Meio Ambiente e dos Recursos Naturais Renováveis, vol. 1, 182p.

Iвама. 2003. Lista das espécies da fauna brasileira ameaçada de extinção. Brasília, Instituto Brasileiro do Meio Ambiente e dos Recursos Naturais Renováveis, Ministério do Meio Ambiente, Instrução Normativa no 3, de 27 de maio de 2003, Diário Oficial da União $n^{\circ}$ 101, ano CXL, seção 1, p. 88-97.

IBge. 1974. Folha Topográfica "Cunha" - SF. 23-Z-C-1-1Ml2771-1. Rio de Janeiro, Instituto Brasileiro de Geografia e Estatística.

Moojen, J. 1952. Os Roedores do Brasil. Rio de Janeiro, Ministério da Educação e Saúde, Instituto Nacional do Livro, 214p.

Musser, G.G.; M.D. Carleton; E.M. Brothers \& A.L. Gardner. 1998. Systematic studies of oryzomyine rodents (Muridae, Sigmodontinae): Diagnoses and distribution of species formerly assigned to Oryzomys "capito". Bulletin of the American Museum of Natural History, New York, 236: 1-376.

OliveIrA, R.F. 1995. Mata Atlântica: Reserva da biodiversidade no Estado do Rio de Janeiro. Revista Feema, Rio de Janeiro, 18: 46-50.

Patton, J.L. \& M.N.F. Da SiLva. 1997. Definition of species of pouched four-eyed opossuns (Didelphidae, Philander). Journal of Mammalogy, Provo, 78: 90-102.

Pereira, L.G; S.E.M. Torres; H.S. Da Silva \& L. Geise. 2001. Nonvolant mammals of Ilha Grande and adjacent areas in southern Rio de Janeiro state, Brazil. Boletim do Museu
Nacional, Nova Série Zoologia, Rio de Janeiro, 459: 1-15. VAZ, S.M. 1998. Primates in the Desengano State Park, Rio de Janeiro, Brazil. Neotropical Primates, Belo Horizonte, 6 (4): 127-128.

Vieira, C.O.O. 1944. Os símios do Estado de São Paulo. Papéis Avulsos do Departamento de Zoologia, São Paulo, 4 (1): $1-31$.

Vieira, C.O.O. 1950. Xenartros e marsupiais do Estado de São Paulo. Arquivos de Zoologia, São Paulo, 5 (3): 325-362.

Vieira, C.O.O. 1955. Lista remissiva dos mamíferos do Brasil. Arquivos de Zoologia, São Paulo, 8 (11):341-474.

Voss, R.S. 1993. A revision of Brazilian muroid rodent genus Delomys with remarks on "Thomasomyine" characters. American Museum Novitates, New York, 3073: 1-44.

Wetzel, R.M. \& E. Mondolfi. 1979. The subgenera and species of long-nosed armadillos, genus Dasypus L., p. 43-63. In: J.F. EISENBERG (Ed.). Vertebrate Ecology in the Northern Neotropics. Washington, Smithsonian Institution Press, 271p.

Wetzel, R.M. \& F.D. Ávila-Pires. 1980. Identification and distribution of the recent sloths of Brazil (Edentata). Revista Brasileira de Biologia, Rio de Janeiro, 40 (4): 831-836.

Wilson, D.E. \& D.M. Reeder. 1993. Mammal species of the world: a taxonomic and geographic reference. Washington, Smithsonian Institution Press, $2^{\text {nd }}$ ed., XVIII+1206p.

Woods, C.A. 1993. Suborder Hystricognathi, p. 771-806. In: D.E. Wilson \& D.M. REEDER (Eds). Mammal species of the world: a taxonomic and geographic reference. Washington, Smithsonian Institution Press, $2^{\text {nd }}$ ed., XVIII+1206p.

Recebido em 17.II.2005; aceito em 01.XII.2005. 